\title{
Conditional Value-at-Risk under ellipsoidal uncertainties
}

\author{
M. H. Wong \\ The Chinese University of Hong Kong, Hong Kong
}

\begin{abstract}
Although Value-at-Risk (VaR) has been widely adapted in financial management, Conditional Value-at-risk (CVaR), which is also known as mean excess loss, mean shortfall, or tail VaR, has also gained importance over the past decade. This is largely owing to the more appealing mathematical properties of the latter. Based on Rockafellar and Uryasev's idea, we are going to look into the CVaR under an ellipsoidal distribution. With the ad-hoc primal-dual interior-point algorithm, we will also focus on the technique that minimizes the CVaR under the framework of portfolio selection.

Keywords: Conditional Value-at-Risk (CVaR), Value-at-Risk (VaR), interior point algorithm, ellipsoidal uncertainties, clustered scenarios, Risk Management, portfolio management.
\end{abstract}

\section{Introduction}

Risk Management plays an important role in assets allocation. It entails the exercise of control over some statistical characteristics of the uncertain portfolio return. Conditional Value-at-risk (CVaR), which is also known as mean excess loss or tail $\mathrm{VaR}$, has gained importance in this aspect over the past decade. This is largely owing to the more appealing mathematical properties compared to the VaR. Based on Rockafellar and Uryasev's [1, 2] idea, we are going to look into the CVaR under the ellipsoidal uncertainties. A portfolio selection problem, using CVaR as the objective, will be discussed in the framework of stochastic optimization. To model the ellipsoidal uncertainties, we will propose a "Clustered Tree", which is deviated from the traditional scenario tree $[3,4,5]$ in the sense that each discretization in the latter is now represented by an ellipsoidal cluster. We will also work out 
the solution method using the ad-hoc primal-dual interior-point algorithm [6] followed by numerical results.

\section{Derive the conditional Value-at-Risk}

Let $f(\phi, r)=-\phi^{T} r$ be our loss function with the portfolio vector $\phi$ and uniform random return vector $r \in \mathcal{E}:=\left\{r \in \mathbf{R}^{n} \mid(r-c)^{T} Q(r-c) \leq \rho^{2}\right\} \subset \mathbf{R}^{n}$. Without loss of generality, we assume $Q$ to be the identity matrix (otherwise, we can do the transformation $\left.\tilde{r}=Q^{\frac{1}{2}}(r-c)+c\right)$ so that $r$ lies on a sphere with a density function $p(r)$ equal to the inverse of its volume, i.e. $p(r):=\frac{1}{\Omega_{n} \rho^{n}}[7]$, where $\Omega_{n}:=\frac{\pi^{\frac{n}{2}}}{\Gamma\left(\frac{n}{2}+1\right)}$. The probability of $f(\phi, r)$ not exceeding a threshold $\alpha$ is then given by

$$
\Psi(\phi, r)=\int_{f(\phi, r) \leq \alpha} p(r) d y .
$$

As a function of $\alpha$ for fixed $\phi, \Psi$ is the cumulative distribution function for the loss associated with $\phi$. It completely determines the behavior of this random variable and is fundamental in defining VaR and CVaR. With a specified probability level $\beta \in(0,1)$, the $\beta$-VaR and $\beta$-CVaR values for the loss random variable associated with $\phi$ are given by:

$$
\begin{gathered}
\operatorname{VaR}_{\beta}(\phi):=\alpha_{\beta}(\phi):=\min \{\alpha \in \mathbf{R} \mid \Psi(\phi, \alpha) \geq \beta\} \\
\operatorname{CVaR}_{\beta}(\phi):=\frac{1}{1-\beta} \int_{f(\phi, r) \geq \alpha_{\beta}(\phi)} f(\phi, r) p(r) d r
\end{gathered}
$$

In the first formula $\alpha_{\beta}(\phi)$ comes out as the left endpoint of the nonempty interval consisting of the values $\alpha$ such that $\Psi(\phi, \alpha)=\beta$, since $\Psi(\phi, \alpha)$ is continuous and nondecreasing with respect to $\alpha$. In the second formula, the probability that $f(\phi, r) \geq \alpha_{\beta}(\phi)$ is therefore equal to $1-\beta$. Hence $C \operatorname{VaR} \beta(\phi)$ comes out as the conditional expectation of the loss associated with $\phi$ relative to that loss being $\alpha_{\beta}(\phi)$ or greater.

Our objective is to minimize the $\beta-C V a R$, but solving this directly seems to be difficult owing to the nature of its definition in terms of the $\beta$-VaR value $\left(\alpha_{\beta}(\phi)\right)$ and the often poor mathematical properties of that value. Fortunately, we can handle a far simpler expression introduced by Rockafellar and Uryasev:

$$
F_{\beta}(\phi, \alpha):=\alpha+\frac{1}{1-\beta} \int_{r \in \mathcal{B}}[f(\phi, r)-\alpha]^{+} p(r) d r .
$$

A crucial feature of $F_{\beta}$ is its joint convexity with respect to $\phi$ and $\alpha$. A very significant result in their paper is that minimizing $C V a R_{\beta}(\phi)$ over the set of possible $\phi$ 's, say $X$, is equivalent to minimizing $F_{\beta}(\phi, \alpha)$ over the set $(\phi, \alpha) \in X \times \mathbf{R}$, i.e.

$$
\min _{\phi \in X} \operatorname{CVa} R_{\beta}(\phi)=\min _{(\phi, \alpha) \in X \times \mathbf{R}} F_{\beta}(\phi, \alpha) .
$$

Since $F_{\beta}(\phi, \alpha)$ is convex with respect to $(\phi, \alpha)$, assuming $X$ to be a convex set, the joint minimization is a convex programming problem. This is a high motivation 
leading us to explore this function in our settings. We now go into the details of the integration in $F_{\beta}$. As $p(r)$ is also a constant, we omit this term for the time being. Consider the case when $\frac{\alpha+\phi^{T} c}{\|\phi\|}<\rho$ :

$$
\begin{aligned}
& \int_{r \in \mathcal{E}}[f(\phi, r)-\alpha]^{+} d r=\int_{r \in \mathcal{E}}\left[-\phi^{T} r-\alpha\right]^{+} d r \\
& =\int_{\substack{(r-c)^{T}(r-c) \leq \rho \\
\phi^{T} r \leq-\alpha}}\left(-\phi^{T} r-\alpha\right) d r \\
& =\int_{\phi^{T} \widetilde{r}^{T} \leq-\phi^{T} c-\alpha}\left(-\phi^{T} \widetilde{r}-\phi^{T} c-\alpha\right) d \widetilde{r} \\
& =\int_{-\rho}^{\frac{-\alpha-\phi^{T} c}{\|\phi\|}}\left(-\|\phi\| t-\phi^{T} c-\alpha\right)\left(\rho^{2}-t^{2}\right)^{\frac{n-1}{2}} d t \\
& =-\left(\phi^{T} c+\alpha\right) \int_{-\rho}^{\frac{-\alpha-\phi^{T} c}{\|\phi\|}}\left(\rho^{2}-t^{2}\right)^{\frac{n-1}{2}} d t \\
& -\|\phi\| \int_{-\rho}^{\frac{-\alpha-\phi^{T} c}{\|\phi\|}} t\left(\rho^{2}-t^{2}\right)^{\frac{n-1}{2}} d t \\
& =-\left(\phi^{T} c+\alpha\right) g_{1}(\phi, \alpha)-\|\phi\| g_{2}(\phi, \alpha),
\end{aligned}
$$

where

$$
\begin{aligned}
& g_{1}(\phi, \alpha)=\int_{-\rho}^{\frac{-\alpha-\phi T_{c}}{\|\phi\|}}\left(\rho^{2}-t^{2}\right)^{\frac{n-1}{2}} d t \\
& g_{2}(\phi, \alpha)=\int_{-\rho}^{\frac{-\alpha-\phi T_{c}}{\|\phi\|}} t\left(\rho^{2}-t^{2}\right)^{\frac{n-1}{2}} d t
\end{aligned}
$$

We writing the constant term as $A_{\beta, n}:=\frac{p(r)}{1-\beta}=\frac{1}{(1-\beta) \Omega_{n} \rho^{n}}$, the function now becomes

$$
F_{\beta}(\phi, \alpha)= \begin{cases}\alpha-A_{\beta, n}\left(\left(\phi^{T} c+\alpha\right) g_{1}(\phi, \alpha)+\|\phi\| g_{2}(\phi, \alpha)\right), & \frac{\alpha+\phi^{T} c}{\|\phi\|}<\rho ; \\ \alpha, & \frac{\alpha+\phi^{T} c}{\|\phi\|} \geq \rho .\end{cases}
$$

where, for $\frac{\alpha+\phi^{T} c}{\|\phi\|}<\rho$,

$$
\begin{aligned}
\nabla_{\phi} g_{1} & =\left(\rho^{2}-\left(\frac{\alpha+\phi^{T} c}{\|\phi\|}\right)^{2}\right)^{\frac{n-1}{2}}\left(-\frac{c}{\|\phi\|}+\left(\alpha+\phi^{T} c\right) \frac{\phi}{\|\phi\|^{3}}\right) \\
\frac{\partial g_{1}}{\partial \alpha} & =-\frac{1}{\|\phi\|}\left(\rho^{2}-\left(\frac{\alpha+\phi^{T} c}{\|\phi\|}\right)^{2}\right)^{\frac{n-1}{2}}
\end{aligned}
$$


220 Computational Finance and its Applications III

$$
\begin{aligned}
\nabla_{\phi} g_{2} & =\frac{-\alpha-\phi^{T} c}{\|\phi\|}\left(\rho^{2}-\left(\frac{\alpha+\phi^{T} c}{\|\phi\|}\right)^{2}\right)^{\frac{n-1}{2}}\left(-\frac{c}{\|\phi\|}+\left(\alpha+\phi^{T} c\right) \frac{\phi}{\|\phi\|^{3}}\right) \\
\frac{\partial g_{2}}{\partial \alpha} & =\frac{\alpha+\phi^{T} c}{\|\phi\|^{2}}\left(\rho^{2}-\left(\frac{\alpha+\phi^{T} c}{\|\phi\|}\right)^{2}\right)^{\frac{n-1}{2}}
\end{aligned}
$$

The above derivatives will be zero otherwise. So, the first and second derivatives are

$$
\begin{aligned}
& \nabla_{\phi} F_{\beta}= \begin{cases}-A_{\beta, n}\left(c g_{1}+\frac{\phi}{\|\phi\|} g_{2}\right), & \frac{\alpha+\phi^{T} c}{\|\phi\|}<\rho \\
0, & \frac{\alpha+\phi^{T} c}{\|\phi\|} \geq \rho .\end{cases} \\
& \frac{\partial F_{\beta}}{\partial \alpha}= \begin{cases}1-A_{\beta, n} g_{1}, & \frac{\alpha+\phi^{T} c}{\|\phi\|}<\rho ; \\
1, & \frac{\alpha+\phi^{T} c}{\|\phi\|} \geq \rho .\end{cases} \\
& \frac{\partial^{2} F_{\beta}}{\partial \phi_{i} \partial \alpha}= \begin{cases}\frac{A_{\beta, n}}{\|\phi\|}\left(\rho^{2}-\left(\frac{\alpha+\phi^{T} c}{\|\phi\|}\right)^{2}\right)^{\frac{n-1}{2}}\left(c_{i}-\frac{\left(\alpha+\phi^{T} c\right) \phi_{i}}{\|\phi\|^{2}}\right), & \frac{\alpha+\phi^{T} c}{\|\phi\|}<\rho ; \\
0, & \frac{\alpha+\phi^{T} c}{\|\phi\|} \geq \rho .\end{cases} \\
& \frac{\partial^{2} F_{\beta}}{\partial \phi_{i}^{2}}= \begin{cases}\frac{A_{\beta, n}}{\|\phi\|}\left[\left(\rho^{2}-\left(\frac{\alpha+\phi^{T} c}{\|\phi\|}\right)^{2}\right)^{\frac{n-1}{2}}\left(c_{i}-\frac{\left(\alpha+\phi^{T} c\right) \phi_{i}}{\|\phi\|^{2}}\right)^{2}\right. & \\
\left.-\left(1-\frac{\phi_{i}^{2}}{\|\phi\|^{2}}\right) g_{2}\right], & \frac{\alpha+\phi^{T} c}{\|\phi\|}<\rho ; \\
0, & \frac{\alpha+\phi^{T} c}{\|\phi\|} \geq \rho .\end{cases} \\
& \frac{\partial^{2} F_{\beta}}{\partial \phi_{i} \partial \phi_{j}}= \begin{cases}\frac{A_{\beta, n}}{\|\phi\|}\left[\left(\rho^{2}-\left(\frac{\alpha+\phi^{T} c}{\|\phi\|}\right)^{2}\right)^{\frac{n-1}{2}}\left(c_{i}-\frac{\left(\alpha+\phi^{T} c\right) \phi_{i}}{\|\phi\|^{2}}\right)\right. \\
\left.\left(c_{j}-\frac{\left(\alpha+\phi^{T} c\right) \phi_{j}}{\|\phi\|^{2}}\right)+\frac{\phi_{i} \phi_{j}}{\|\phi\|^{2}} g_{2}\right], & \frac{\alpha+\phi^{T} c}{\|\phi\|}<\rho ; \\
0, & \frac{\alpha+\phi^{T} c}{\|\phi\|} \geq \rho,\end{cases} \\
& \forall i \neq j
\end{aligned}
$$




\section{Single cluster model and numerical algorithm}

As the usual portfolio selection model, we would consider the constraint set which includes no shorting selling, meeting (at least) the target return $R$, and the budget constraint. By (3) and (4), the model of minimizing the $\beta-C V a R$ becomes

$$
\begin{array}{ll}
\min & F_{\beta}(\phi, \alpha) \\
\text { s.t. } & \phi^{T} e=1 \\
& \phi^{T} c \geq R \\
& \phi \geq 0
\end{array}
$$

We will use the interior point algorithm again to solve the problem. For convenience, we add the slack variables $\phi_{n+1}, s:=\left(s_{1}, \cdots, s_{n}\right)^{T}$ to the inequalities and obtain:

$$
\begin{array}{r}
\phi^{T} c-R-\phi_{n+1}=0 \\
-\phi+s=0 .
\end{array}
$$

The Langrangian function is given as

$$
L(x, \alpha, y, z)=F_{\beta}(\phi, \alpha)+y_{1}\left(\phi^{T} e-1\right)+y_{2}\left(\phi^{T} c-R-\phi_{n+1}\right)-z^{T} x
$$

where $x=\left(\begin{array}{c}\phi \\ \phi_{n+1}\end{array}\right) \in \mathbf{R}^{n+1}, y=\left(\begin{array}{c}y_{1} \\ y_{2}\end{array}\right) \in \mathbf{R}^{2}$, and $z=\left(\begin{array}{c}z^{\prime} \\ z_{n+1}\end{array}\right)=$ $\left(\begin{array}{c}z_{1} \\ \vdots \\ z_{n} \\ z_{n+1}\end{array}\right) \in \mathbf{R}^{n+1}$

The first order KKT conditions are

$$
\begin{aligned}
\nabla_{\phi} F_{\beta}(\phi, \alpha)+y_{1} e+y_{2} c-z & =0 \\
\left(\nabla_{\alpha} F_{\beta}(\phi, \alpha)=\right) 1-A_{\beta, n} g_{1}(\phi, \alpha) & =0 \\
\left(\nabla_{\phi_{n+1}} L(x, \alpha, y, x)=\right)-y_{2}-z_{n+1} & =0 \\
\phi^{T} e-1 & =0 \\
\phi^{T} c-R-\phi_{n+1} & =0 \\
-x+s & =0 \\
z_{i} s_{i} & =0, \quad i=1, \cdots, n+1 \\
(z, s) & \geq 0
\end{aligned}
$$

Writing the no-shorting selling constraints (with the slack variable $\phi_{n+1}$ ) as a vector function $h(x)=-x$, the Jacobian of it will be $J_{h}(x)=-I_{(n+1) \times(n+1)}$. Hence, 
the modified Newton step used for interior-point algorithm for the equality conditions becomes

$$
\begin{gathered}
\left(\begin{array}{ccccc}
\nabla_{x}^{2} L & \nabla_{\alpha}\left(\nabla_{x} L\right) & \nabla_{y}\left(\nabla_{x} L\right) & J_{h}(x)^{T} & 0_{(n+1) \times(n+1)} \\
\nabla_{x}\left(\nabla_{\alpha} L\right) & \nabla_{\alpha}^{2} L & \nabla_{y}\left(\nabla_{\alpha} L\right) & 0_{2 \times(n+1)} & 0_{2 \times(n+1)} \\
\nabla_{x}\left(\nabla_{y} L\right) & 0_{2 \times 1} & \nabla_{y}^{2} L & 0_{2 \times(n+1)} & 0_{2 \times(n+1)} \\
J_{h}(x) & 0_{(n+1) \times 1} & 0_{(n+1) \times 2} & 0_{(n+1) \times(n+1)} & I_{(n+1) \times(n+1)} \\
0_{(n+1) \times(n+1)} & 0_{(n+1) \times 1} & 0_{(n+1) \times 2} & S & Z
\end{array}\right)\left(\begin{array}{c}
\Delta x \\
\Delta \alpha \\
\Delta y \\
\Delta z \\
\Delta s
\end{array}\right) \\
=\left(\begin{array}{c}
-\nabla_{x} L \\
-\nabla_{\alpha} L \\
-\nabla_{y} L \\
-(h(x)+s) \\
-S Z e+\sigma \mu e
\end{array}\right)
\end{gathered}
$$

where

$$
\text { - } S=\left(\begin{array}{ccc}
s_{1} & & \\
& \ddots & \\
& & s_{n+1}
\end{array}\right), Z=\left(\begin{array}{ccc}
z_{1} & & \\
& \ddots & \\
& & z_{n+1}
\end{array}\right) \text {, }
$$

- $\mu=\frac{s^{T} z}{n+1}, \sigma \in(0,1)$ is arbitrarily chosen,

- $\nabla_{\alpha}\left(\nabla_{x} L(x, \alpha, y, z)\right)=\left(\begin{array}{c}\frac{\partial^{2} F_{\beta}}{\partial \phi_{1} \partial \alpha} \\ \vdots \\ \frac{\partial^{2} F_{\beta}}{\partial \phi_{n} \partial \alpha} \\ 0\end{array}\right)=\nabla_{x}\left(\nabla_{\alpha} L(x, \alpha, y, z)\right)^{T}$,

- $\nabla_{\alpha}^{2} L(x, \alpha, y, z)=\frac{\partial^{2} F_{\beta}}{\partial \alpha^{2}}$

- $\nabla_{y}\left(\nabla_{\alpha} L(x, \alpha, y, z)\right)=0_{1 \times 2}=\nabla_{\alpha}\left(\nabla_{y} L(x, \alpha, y, z)\right)^{T}$,

- $\nabla_{y}\left(\nabla_{x} L(x, \alpha, y, z)\right)=\left(\begin{array}{cc}1_{n \times 1} & c \\ 0 & -1\end{array}\right)=\left(\nabla_{x}\left(\nabla_{y} L(x, \alpha, y, z)\right)\right)^{T}$,

- $\nabla_{x}^{2} L(x, \alpha, y, z)=\left(\begin{array}{cc}\nabla_{\phi}^{2} F_{S D}(\phi) & 0_{n \times 1} \\ 0_{n \times 1} & 0\end{array}\right)$,

and $\nabla_{y}^{2} L(x, \alpha, y, z)=0_{2 \times 2}$

\section{Multi-clusters model}

We now go to explore the multiple clusters model. Suppose there are $m$ clusters. Our return $r$ could now be $r^{(k)}$ with a probability of $\omega^{(k)}$ and whose expected return are $c^{(k)}$ for $j=1, \cdots, m$. In other words, each $r^{(k)}$ is in an ellipsoid $\mathcal{E}^{(k)}:=\{r \in$ $\left.\mathbf{R}^{n} \mid\left(r-c^{(k)}\right)^{T} Q^{(k)}\left(r-c^{(k)}\right) \leq\left(\rho^{(k)}\right)^{2}\right\}$. Again, for simplicity, we assume all $Q^{(k)}$ 's are identity matrices and all $\rho^{(k)}$ 's are equal to $\rho$. So, the clusters are now 
only differed by the centres $c^{(k)}$ 's. We would also consider a function like (4) in order to minimize the $C V a R$. In fact, it is a weighted average of (4). Let

$$
\begin{aligned}
& F_{\beta}^{(k)}(\phi, \alpha) \\
& := \begin{cases}\alpha-A_{\beta, n}\left(\left(\phi^{T} c^{(k)}+\alpha\right) g_{1}^{(k)}(\phi, \alpha)+\|\phi\| g_{2}^{(k)}(\phi, \alpha)\right), & \frac{\alpha+\phi^{T} c}{\|\phi\|}<\rho ; \\
\alpha, & \frac{\alpha+\phi^{T} c}{\|\phi\|} \geq \rho,\end{cases}
\end{aligned}
$$

where $g_{1}^{(k)}$ and $g_{2}^{(k)}$ are the same as $g_{1}$ and $g_{2}$ respectively except that $c$ is replaced by $c^{(k)}$. Then our objective function for $\frac{\alpha+\phi^{T} c}{\|\phi\|}<\rho$ is derived as follows.

$$
\begin{aligned}
F_{M C}: & =\sum_{k=1}^{m} \omega^{(k)} F_{\beta}^{(k)} \\
& =\alpha+\frac{1}{1-\beta} \sum_{k=1}^{m} \omega^{(k)} \int_{r \in \mathcal{E}^{(k)}}[f(\phi, r)-\alpha]^{+} p(r) d r \\
& =\alpha+A_{\beta, n} \sum_{k=1}^{m} \omega^{(k)} \int_{r \in \mathcal{E}^{(k)}}\left[-\phi^{T} r-\alpha\right]^{+} d r \\
& =\alpha+A_{\beta, n} \sum_{k=1}^{m} \omega^{(k)} \int_{-\rho}^{\frac{-\alpha-\phi^{T} c^{(k)}}{\|\phi\|}}\left(-\|\phi\| t-\phi^{T} c^{(k)}-\alpha\right)\left(\rho^{2}-t^{2}\right)^{\frac{n-1}{2}} d t \\
& =\alpha-A_{\beta, n} \sum_{k=1}^{m} \omega^{(k)}\left[\left(\phi^{T} c^{(k)}+\alpha\right) g_{1}^{(k)}(\phi, \alpha)+\|\phi\| g_{2}^{(k)}(\phi, \alpha)\right]
\end{aligned}
$$

with the derivatives

$$
\begin{aligned}
\frac{\partial F_{M C}}{\partial \phi} & =-A_{\beta, n} \sum_{k=1}^{m} \omega^{(k)}\left(c g_{1}^{(k)}+\frac{\phi}{\|\phi\|} g_{2}^{(k)}\right) \\
\frac{\partial F_{M C}}{\partial \alpha} & =1-A_{\beta, n} \sum_{k=1}^{m} \omega_{k} g_{1}^{(k)} \\
\frac{\partial^{2} F_{M C}}{\partial \phi_{i} \partial \alpha} & =-A_{\beta, n} \sum_{k=1}^{m} \omega^{(k)} \frac{\partial g_{1}^{(k)}}{\partial \phi_{i}} \\
& =\frac{A_{\beta, n}}{\|\phi\|} \sum_{k=1}^{m} \omega^{(k)}\left(\rho^{2}-\left(\frac{\alpha+\phi^{T} c^{(k)}}{\|\phi\|}\right)^{2}\right)^{\frac{n-1}{2}}\left(c_{i}^{(k)}-\frac{\left(\alpha+\phi^{T} c\right) \phi_{i}}{\|\phi\|^{2}}\right)
\end{aligned}
$$




$$
\begin{aligned}
\frac{\partial^{2} F_{M C}}{\partial \phi_{i}^{2}}= & \frac{A_{\beta, n}}{\|\phi\|} \sum_{k=1}^{m} \omega^{(k)}\left[\left(\rho^{2}-\left(\frac{\alpha+\phi^{T} c^{(k)}}{\|\phi\|}\right)^{2}\right)^{\frac{n-1}{2}}\left(c_{i}^{(k)}-\frac{\left(\alpha+\phi^{T} c^{(k)}\right) \phi_{i}}{\|\phi\|^{2}}\right)^{2}\right. \\
& \left.-\left(1-\frac{\phi_{i}^{2}}{\|\phi\|^{2}}\right) g_{2}^{(k)}\right] \\
\frac{\partial^{2} F_{M C}}{\partial \phi_{i} \partial \phi_{j}}= & \frac{A_{\beta, n}}{\|\phi\|} \sum_{k=1}^{m} \omega^{(k)}\left[\left(\rho^{2}-\left(\frac{\alpha+\phi^{T} c^{(k)}}{\|\phi\|}\right)^{2}\right)^{\frac{n-1}{2}}\right. \\
& \left.\times\left(c_{i}^{(k)}-\frac{\left(\alpha+\phi^{T} c^{(k)}\right) \phi_{i}}{\|\phi\|^{2}}\right)\left(c_{j}^{(k)}-\frac{\left(\alpha+\phi^{T} c^{(k)}\right) \phi_{j}}{\|\phi\|^{2}}\right)+\frac{\phi_{i} \phi_{j}}{\|\phi\|^{2}} g_{2}^{(k)}\right] \\
& \forall i \neq j .
\end{aligned}
$$

We can see that our objective function is a linear combination of convex functions $\left(F_{\beta}^{(k)}\right)$, which is still convex. Hence we can apply the same techniques as before to solve the model:

$$
\begin{array}{ll}
\min & F_{M C}(\phi) \\
\text { s.t. } & \phi^{T} e=1 \\
& \phi^{T}\left(\sum_{k=1}^{m} \omega^{(k)} c^{(k)}\right) \geq R \\
& \phi \geq 0
\end{array}
$$

In particular, the numerical procedure will be only differed by the derivatives such that those of $F_{\beta}$ is now replaced by those of $F_{M C}$, and the explicit forms are already shown above.

As a reminder, it is worth mentioning that the corresponding $\beta$-VaR value (the optimal value of $\alpha$ ) comes out as a by-product of the optimization of $\beta$ - $C V a R$, but this optimal value $\alpha$ is not equivalent to the result of minimizing $\alpha_{\beta}(\phi)$ directly over the same constraint set. However, since $\beta-C \operatorname{VaR}(\phi) \geq \beta$-VaR $(\phi)$, solutions to our problem should also be good from the perspective of minimizing $\alpha_{\beta}(\phi)$. We will go through the numerical results in the next session.

\section{Numerical results}

Suppose there are three scenarios in next stage. We will assume they are all spheres instead of ellipsoids. Here are the expected rates of return for three stocks in each of the cluster. In other words, these are the centres of the spheres. We set a radius of 0.4 for each.

\begin{tabular}{|c|c|c|c|}
\hline Stocks \Scenarios & 1 & 2 & 3 \\
\hline $\mathrm{A}$ & 2 & 1.2 & 0.8 \\
$\mathrm{~B}$ & 1.8 & 1.3 & 0.7 \\
$\mathrm{C}$ & 0.5 & 1.2 & 1.9 \\
\hline
\end{tabular}


Table 1: Numerical results for the CVaR model.

\begin{tabular}{|c|r|r|r|r|}
\hline$\beta$ & \multicolumn{1}{|c|}{0.85} & \multicolumn{1}{c|}{0.90} & \multicolumn{1}{c|}{0.95} & \multicolumn{1}{c|}{0.99} \\
\hline portfolio & 0.296568668 & 0.286307431 & 0.274452573 & 0.250280625 \\
& 0.281468101 & 0.287049666 & 0.293294229 & 0.310341706 \\
& 0.421963231 & 0.426642903 & 0.432253197 & 0.439377668 \\
$E\left[\phi^{T} r\right]$ & 1.250766974 & 1.250159468 & 1.249442324 & 1.248303690 \\
$C V a R$ & -1.130975111 & -1.092842583 & -1.046622223 & -0.987803416 \\
$V a R$ & -1.239149005 & -1.174644069 & -1.100725596 & -1.011797682 \\
\hline
\end{tabular}

We may regard cluster 1 and 3 as the two extreme states of the economy, while cluster 2 may be regarded as a stable economy. In this sense, we assign a probability of occurrence arbitrarily to each state as follows.

\begin{tabular}{|c|c|c|c|}
\hline Cluster & 1 & 2 & 3 \\
\hline Probability & $\frac{1}{4}$ & $\frac{1}{2}$ & $\frac{1}{4}$ \\
\hline
\end{tabular}

Applying our notations, we will have the following:

- $c^{(1)}=(2,1.8,0.5)^{T}$

- $c^{(2)}=(1.2,1.3,1.2)^{T}$

- $c^{(3)}=(0.8,0.7,1.9)^{T}$

- $\rho^{(k)}=\rho=0.5 \quad \forall \quad k=1,2,3$

- $\omega^{(1)}=.25$

- $\omega^{(2)}=.5$

- $\omega^{(3)}=.25$

Using the primal-dual interior-point algorithm with the modified Newton step shown in Section 3, we can solve our model efficiently. By varying the threshold parameter $\beta$ representing the confidence level, the numerical results are shown in Table 1.

Agreeing with the model, the result shows that $R$ is almost an independent factor unless it reaches its the critical value (1.3) or a higher level of confidence $(\beta>0.9)$ is of our concern. Also, the $C V a R$ as well as the associated $V a R$ is decreasing as we increases our confidence level $\beta$, which is in line with our common sense that risk has to be eliminated for a greater certainty (or $\beta$ ).

\section{Conclusion}

After investigating into the numerical results, we may conclude that the CVaR model is reasonably practical in the sense that the numerical results meet our intuition. As a matter of fact, some other measured can also be derived based on our clustered tree, for instance, the downside risk measure and the probability of a certain level of attainment. The close form solutions are also as nice as the CVaR. 
However, there is a hinderance for our clustered tree to develop further into a two-stage tree. The main challenge lies on the recourse problem in the stochastic programming, in which we will suffer from an infinite number of constraints.

Some further directions of this area could be trying to alternate the distribution from the uniform one to others. A step density function that cumulates more "mass" towards the center of the ellipsoid may be considered. Meanwhile, we may also extend our discussion to a broader class of distributions, namely, the radial distribution. If this extension is successful, it may result in a wider application of our tree, since the commonly used Gaussian distribution also falls into this class. An artificial data set is created for the numerical tests instead of data set that generated from proper sampling techniques because we have failed to find a suitable set of sample data so far. Therefore, searching for a set of proper real market data for the implementation is also an important issue.

\section{References}

[1] Rockafellar, R. \& Uryasev, S., Optimization of conditional value-at-risk. Journal of Risk, 2, 2000.

[2] Rockafellar, R. \& Uryasev, S., Coniditional value-at-risk for general loss distributions. Journal of Banking and Finance, 26, 2002.

[3] Kaut, M. \& Wallace, W., Evaluation of scenario-generation methods for stochastic programming. Stochastic Programming E-Print Series (ispep$\left.\operatorname{sorg}_{6}\right),(\mathbf{1 4}), 2003$.

[4] Kouwenbery, R., Scenario generation and stochastic programming models for asset liability management. European Journal of Operational Research, 134, 2001.

[5] Hoyland, K. \& Wallace, W., Generating scenario trees for multistage decision problems. Management Science, 47, 2001.

[6] Wright, S.J., Primal-dual interior-point methods. Philadelphia : Society for Industrial and Applied Mathematics, 1997.

[7] Hotelling, H., Tubes and spheres in n-spaces, and a class of statistical problems. American Journal of Mathematics, 61(2). 